\title{
RESEARCH
}

\section{Falsely elevated point-of-care lactate measurement after ingestion of ethylene glycol}

\author{
Peter G. Brindley, Matthew S. Butler, George Cembrowski, David N. Brindley
}

\section{ABSTRACT}

Background: A patient presented with severe acidosis and a point-of-care lactate measurement of $42 \mathrm{mmol} / \mathrm{L}$. Mesenteric ischemia was suspected, with a potential need for laparotomy; however, plasma lactate measurements were below $4 \mathrm{mmol} / \mathrm{L}$. Ethylene glycol ingestion was subsequently diagnosed. We therefore wished to determine why discrepancies in lactate measurements occur and whether this "lactate gap" could be clinically useful.

Methods: We phlebotomized blood, added various concentrations of metabolites of ethylene glycol, and tested the resulting samples with the 5 most common lactate analyzers.

Results: With the Radiometer 700 point-of-care analyzer, glycolate addition resulted in an artifactual, massive lactate elevation, even at low glycolate concentrations. Another major ethylene glycol metabolite, glyoxylate (but not oxalate or formate), caused similar elevations. The i-STAT and Bayer point-of-care analyzers and the Beckman and Vitros laboratory analyzers reported minimal lactate elevations. Lactate gap was determined by comparing the Radiometer result with the corresponding result from any of the other analyzers.

Interpretation: We demonstrated how inappropriate laparotomy or delayed therapy might occur if clinicians are unaware of this phenomenon or have access to only a single analyzer. We also showed that lactate gap can be exploited to expedite treatment, diagnose late ethylene-glycol ingestion and terminate dialysis. By comparing lactate results from the iSTAT or Bayer devices with that from the Radiometer, ethylene-glycol ingestion can be diagnosed at the point of care. This can expedite diagnosis and treatment by hours, compared with waiting for laboratory results for plasma ethylene glycol.

CMAJ 2007;176(8):1097-9

49-year-old woman was found at home by her family with decreased consciousness of unknown cause. En route to the emergency department, paramedics intubated her because of poor airway support and evolving multisystem organ failure. Point-of-care testing in the emergency department of arterial blood indicated severe acidosis $(\mathrm{pH}$
< 6.9) with a lactate concentration of $42 \mathrm{mmol} / \mathrm{L}$ (Table I). Little other medical history was available at that time.

She was transferred to the hospital's intensive care unit. A general surgeon was consulted in case the lactate elevation represented severe mesenteric ischemia requiring urgent laparotomy. Use of sedatives and neuromuscular blocking medications precluded an adequate abdominal examination. Although family consent was obtained for immediate laparotomy, we delayed to obtain an abdominal CT scan and to improve her hemodynamics and reduce her acidosis. Blood work at admission included a laboratory plasma-lactate measurement, for which a sample was drawn minutes after the arterial blood-gas sample.

Unexpectedly, the plasma lactate concentration was only I. $5 \mathrm{mmol} / \mathrm{L}$.

Two hours later, full laboratory investigations showed an increased serum osmolarity $(353 \mathrm{mmol} / \mathrm{L})$ and an osmolar gap of $33 \mathrm{mmol} / \mathrm{L}$. Urine analysis found calcium oxalate crystals. An urgent ethylene glycol test showed a plasma concentration of $15 \mathrm{mmol} / \mathrm{L}$. Resuscitation continued, but now included high-flux dialysis and an intravenous infusion of

Table 1: Point-of-care arterial blood gas and laboratory (plasma) values at various times of day in the case reported

\begin{tabular}{lcccc}
\hline Measurement & $07: 20$ & $09: 10$ & $14: 50$ & $21: 40$ \\
\hline Lactate, mmol/L & & & & \\
\hline Arterial blood gas* & 42 & 36 & 29 & 18 \\
\hline Plasma $†$ & $<1.5$ & 2.0 & 2.8 & 3.6 \\
\hline Ethylene glycol, mmol/L & - & 15 & 8 & 4 \\
\hline Ethanol,‡ mmol/L & - & $<3$ & 27 & 40 \\
\hline pH $^{*}$ & 6.88 & 6.81 & 7.02 & 7.37 \\
\hline Base excess* & -25.2 & -24.2 & -22 & -6 \\
\hline Osmolar gap,§ mmol/L & 48 & 39 & 30 & - \\
\hline
\end{tabular}

*Measured with a Radiometer ABL 700 point-of-care whole-blood analyzer. † Measured with a Beckman Coulter LX20 plasma automated analyzer. ‡Intravenous infusion initiated at 09:20 h, after ethylene glycol ingestion was diagnosed, in keeping with the treatment protocol.

§Determined by the difference between the laboratory measurement (freezing-point osmometry) and the calculated value: $(2 \times \mathrm{Na})+\mathrm{BUN}+$ glucose (where the concentrations of sodium, blood urea nitrogen and glucose are measured in identical units). 
ethanol to counter ethylene glycol. Repeated analysis confirmed high point-of-care readings for lactate and comparatively low plasma-lactate results. Dialysis rapidly decreased the ethylene glycol level, the anion-gap metabolic acidosis, and the lactate measured with the Radiometer point-of-care analyzer. Laboratory readings for plasma lactate never exceeded $3.6 \mathrm{mmol} / \mathrm{L}$ (Table I). The patient's abdominal CT scan was judged normal; no surgery was performed.

The patient was discharged after 2 weeks with residual renal failure requiring intermittent hemodialysis. She admitted to deliberate ingestion of ethylene glycol.

This case demonstrates how misdiagnosis, including inappropriate laparotomy and delayed ethylene-glycol therapy, could occur. We wished to determine why discrepant lactate values occurred, but also if this "lactate gap" "might also be used to expedite diagnosis and therapy.

\section{Methods}

Evidence has suggested that certain point-of-care analyzers might report artifactual (false, machine-generated) lactate elevations as a result of ethylene glycol ingestion. ${ }^{2-4}$ Therefore, 2 of the authors (PGB, DNB) donated venous blood to be phlebotomized into Vacutainers (Becton Dickinson, Singapore) containing EDTA-F (i.e., ethylenedinitrilotetraacetatefluoride) for the Beckman Coulter LX 20 and Vitros laboratory analyzers, or heparin for the i-STAT, Bayer Rapidlab 865 and Radiometer ABL 700 point-of-care analyzers. Samples were quickly pipetted and mixed in equivalent collection tubes. These contained glycolic, glyoxylic, oxalic or formic acid (Sigma-Aldrich, St. Louis, Mo.) to achieve the concentrations desired. These samples were rapidly analyzed with the $5 \mathrm{ma}-$ chines, all of which are common among rural as well as urban hospitals. The University of Alberta Health Research Ethics Board approved this work.

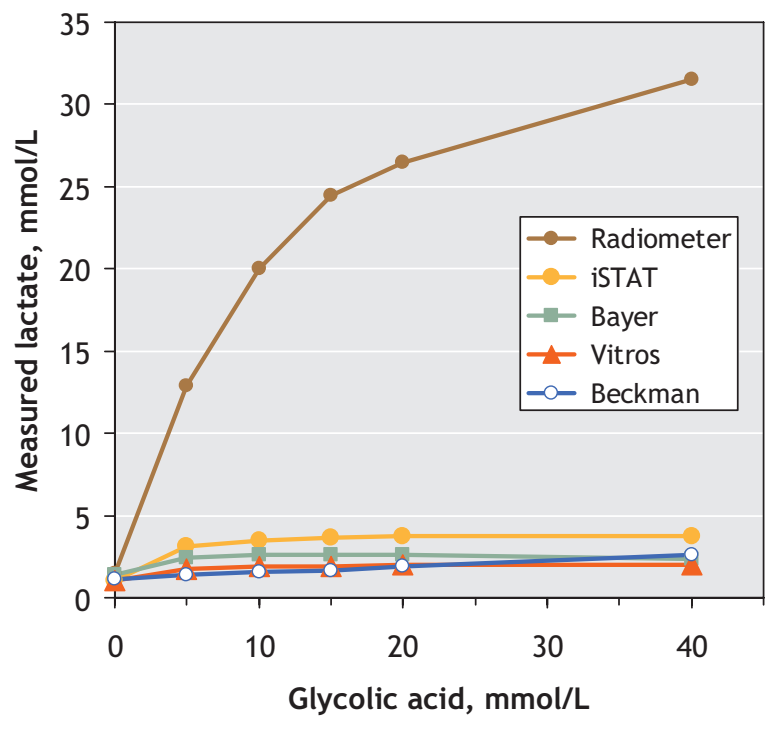

Fig. 1: Blood lactate measurements as a function of glycolate concentration, as measured with 5 commonly used analyzers.

\section{Results}

The Radiometer whole blood gas point-of-care analyzer showed markedly elevated lactate levels, even at a glycolate concentration of only $5 \mathrm{mmol} / \mathrm{L}$ (Fig. I). The iSTAT and Bayer point-ofcare analyzers and the 2 laboratory plasma-analyzers showed minimal elevations $(\leq 4 \mathrm{mmol} / \mathrm{L})$ even at $40 \mathrm{mmol} / \mathrm{L}$ glycolate. We calculated lactate gaps by comparing lactate readings from the Radiometer with those of any of the other 4 analyzers.

Glycolate has previously been shown to cause artifactual lactate elevation, ${ }^{2-4}$ but here we demonstrated that glyoxylate, a predominant human ethylene glycol metabolite, ${ }^{5}$ also does this (Fig. 2). When we added $20 \mathrm{mmol} / \mathrm{L}$ glyoxlate plus $20 \mathrm{mmol} / \mathrm{L}$ glycolate, the apparent lactate concentrate rose to 3I $\mathrm{mmol} / \mathrm{L}$, similar to that after $40 \mathrm{mmol} / \mathrm{L}$ glycolate. Neither oxalate nor formate, the primary methanol metabolite, altered the lactate readings.

Weeks later, a second patient arrived with decreased consciousness and discrepant lactate readings: the point-of-care result was elevated, whereas the laboratory lactate measurement was within the normal range. Rather than wait for laboratory confirmation of ethylene glycol poisoning, we initiated an ethanol infusion and dialysis on the basis of this lactate gap -2 hours before laboratory testing confirmed ethylene glycol. This patient likewise subsequently admitted to ethylene glycol ingestion. In contrast to our first patient, he was discharged within 2 days at baseline health.

Both of the patients who poisoned themselves with ethylene glycol agreed to be described in this publication, on condition of anonymity.

\section{Interpretation}

Our first case illustrated how false-positive lactate might have led to misdiagnosis, inappropriate laparotomy and delayed

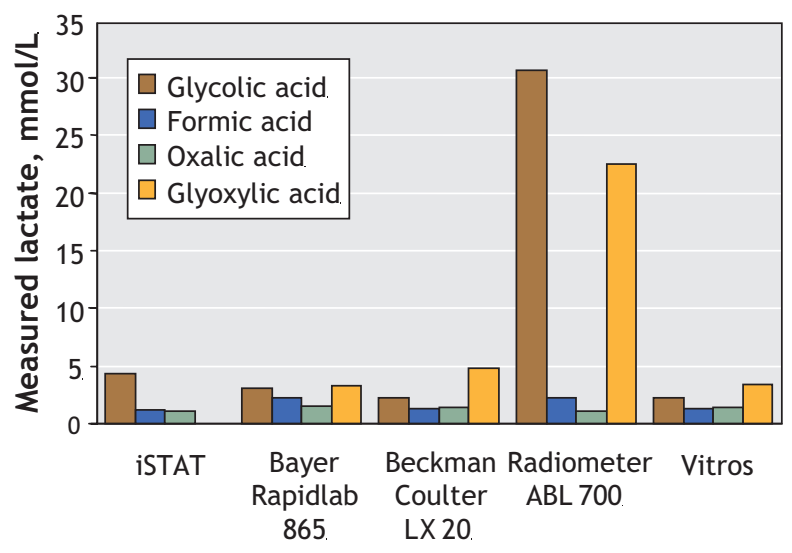

\section{Analyzers}

Fig. 2: The effect of ethylene glycol metabolites (and formate) upon blood lactate concentrations, as measured by 5 common analyzers. The blood concentrations used were $20 \mathrm{mmol} / \mathrm{L}$ of glycolic, glyoxylic, formic or oxalic acid. Note that the iSTAT Analyzer was unable to read glyoxylic acid. 
treatment. The treatment of ethylene glycol ingestion is timedependant; delays could lead to serious consequences, including renal failure and death. Equally, laparotomy, in a patient with severe acidosis, could be associated with considerable perioperative risk. In contrast, our second case demonstrated how discrepant lactate readings can instead be used as a diagnostic clue to actually expedite diagnosis and treatment.

Our experiment revealed false-positive lactate elevation readings for 2 major ethylene glycol metabolites, glycolate and glyoxalate, but only with the Radiometer Analyzer. We have shown how results from the Radiometer that contrast with those from either the i-STAT or Bayer point-of-care analyzers can be used rapidly at the bedside, hours ahead of confirmatory laboratory results, to indicate ethylene glycol poisoning.

Most L-lactate analyzers use L-lactate oxidase. The falsepositive results from the Radiometer likely occur because ethylene glycol metabolites cross-react with L-lactate oxidase. ${ }^{2-4}$ In contrast, ethylene glycol metabolites cause minimal lactate elevation with the Bayer, iSTAT and Vitros devices, to a degree unlikely to cause misdiagnosis. Of note is that, in addition to antifreeze, ethylene glycol is found in many common products. ${ }^{6}$ Our experiment showed that even modest ingestion (causing glycolate and glyoxylate concentrations in blood of 5-Io $\mathrm{mmol} / \mathrm{L}$ ) could produce false-positive lactate results of Io-20 mmol/L with the Radiometer Analyzer (Fig. 2).

Patients who come to hospital late after ethylene glycol ingestion are among the sickest from high metabolite concentrations. However, they are difficult to confirm diagnostically because plasma ethylene glycol concentration and osmolar gap may become normal upon metabolism. Most hospitals measure ethylene glycol, not its metabolites. The lactate gap offers a surrogate test for metabolites (glyoxalate and glycolate) that is useful in diagnosis late presentations. Futhermore, most dialysis protocols rely upon serial measurements of plasma concentrations of ethylene glycol, even though its toxicity results from its metabolites. As long as a lactate gap exists, metabolites still exist. Treatment with dialysis could be ended with confidence, once the gap has disappeared.

Lactate is key in the diagnosis of mesenteric ischemia, and in decisions about laparotomy, because of a sensitivity approaching $100 \%$ (and despite specificities of $42 \%-87 \%$ ). The benefits of point-of-care analyzers include rapid turnaround and low cost. Point-of-care lactate testing is therefore increasingly common, and the Radiometer Analyzer is popular. The potential for misdiagnosis is therefore substantial, especially since patients may deny ethylene-glycol ingestion; such denials might not be doubted, given an elevated lactate result. ${ }^{9,10}$ Clinicians know that quantitative laboratory errors occur, and will therefore repeat suspicious values. However, artifactual errors that require retesting with a different machine are less commonly appreciated.

The lactate gap $^{1}$ is a quick and sensitive test that can be established at the point of care. We showed how a false-positive point-of-care lactate could mean serious misdiagnosis. However, we also showed this to be a diagnostic aid to expedite faster treatment. In short, clinicians should "Mind the Gap."

This article has been peer reviewed.

From the Divisions of Critical Care Medicine (P.G. Brindley) and Medical Biochemistry (Cembrowski), Departments of Surgery (Butler) and Biochemistry (D.N. Brindley), University of Alberta, Edmonton, Alta.

Competing interests: None declared by Peter Brindley, David Brindley and Matthew Butler. George Cembrowski owns stocks in and has accepted fees and travel assistance from companies that make or sell analyzers.

Contributors: All authors were involved in the conception and design of the study as well as the acquisition, analysis and interpretation of the data. All authors drafted and revised the article and approved the final version.

Acknowledgements: We appreciate the contributions of Don LeGatt, Mark Ewanchuk, Julie Mitchell and Jay Dewald.

This study was supported financially by an Open Operating Grant from the Canadian Institutes of Health Research (MOP 4949I).

\section{REFERENCES}

I. Shirey T, Sivilotti M. Reaction of lactate electrodes to glycolate. Crit Care Med I999;27:2305-7.

2. Morgan TJ, Clark C, Clague A. Artifactual elevation of measured plasma L-lactate concentration in the presence of glycolate. Crit Care Med I999;27:2177-9.

3. Woo MY, Greenway DC, Nadler SP, et al. Artifactual elevation of lactate in ethylene glycol poisoning. J Emerg Med 2003;25:289-93.

4. Porter WH, Crellin M, Rutter PW, et al. Interference by glycolic acid in the Beckman synchron method for lactate: a useful clue for unsuspected ethylene glycol intoxication. Clin Chem 2000;46:874-5.

5. Booth ED, Dofferhoff O, Boogaard PJ, et al. Comparison of the metabolism of ethylene glycol and glycolic acid in vitro by precision-cut tissue slices from female rat, rabbit and human liver. Xenobiotica 2004;34:31-48.

6. National Institutes of Health; National Library of Medicine, Specialized Information Services. Ethylene glycol. Household products database. Available: http: //householdproducts.nlm.nih.gov/cgi-bin/household/brands?tbl=chem\&id=I93 (accessed 2007 Feb I)

7. Murray MJ, Gonze MD, Nowak LR, et al. Serum D-lactate levels as an aid to diagnosing acute intestinal ischemia. Am J Surg 1994;167:575-8.

8. Lange $\mathrm{H}$, Jackel $\mathrm{R}$. Usefulness of plasma lactate concentration in the diagnosis of acute abdominal disease. Eur J Surg 1994;160:38I-4.

9. Eder AF, McGrath CM, Dowdy YG, et al. Ethylene glycol poisoning: toxicokinetic and analytical factors affecting laboratory diagnosis. Clin Chem I998;44:I68-77.

Io. Litovitz TL, Smilkstein M, Felberg L, et al. 1996 annual report of the American Association of Poison Control Centers Toxic Exposure Surveillance System. Am J Emerg Med 1997;15:447-500.

Correspondence to: Dr. Peter Brindley, Program Director Critical Care Medicine, 4 H1.22 University of Alberta Hospital, 8840-112th St., Edmonton AB T6G 2B7; fax 780 407-6018; peterbrindley@cha.ab.ca 